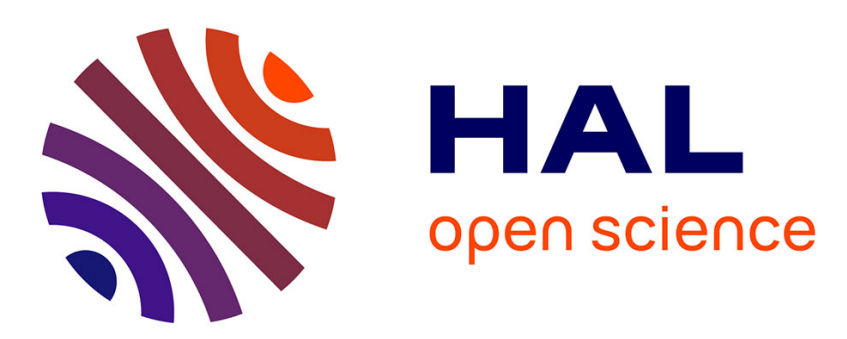

\title{
The role of protein disulphide isomerase AGR2 in the tumour niche
}

Frederic Delom, Amal Nazaraliyev, Delphine Fessart

\section{To cite this version:}

Frederic Delom, Amal Nazaraliyev, Delphine Fessart. The role of protein disulphide isomerase AGR2 in the tumour niche. Biology of the Cell, 2018, 110 (12), pp.271-282. 10.1111/boc.201800024 . hal01940306

\section{HAL Id: hal-01940306 https://hal-univ-rennes1.archives-ouvertes.fr/hal-01940306}

Submitted on 14 Dec 2018

HAL is a multi-disciplinary open access archive for the deposit and dissemination of scientific research documents, whether they are published or not. The documents may come from teaching and research institutions in France or abroad, or from public or private research centers.
L'archive ouverte pluridisciplinaire HAL, est destinée au dépôt et à la diffusion de documents scientifiques de niveau recherche, publiés ou non, émanant des établissements d'enseignement et de recherche français ou étrangers, des laboratoires publics ou privés. 


\author{
Delom F. ${ }^{1,2}$, Nazaraliyev A. ${ }^{1,2}$ and Fessart D. ${ }^{3,4^{*}}$
}

${ }^{1}$ Univ. Bordeaux, INSERM U1218, F-33000 Bordeaux, France.

${ }^{2}$ Institut Bergonié, Comprehensive Cancer Centre, F- 33076 Bordeaux, France

${ }^{3}$ INSERM U1242, "Chemistry, Oncogenesis, Stress, Signaling", Université de Rennes 1, Rennes, France.

${ }^{4}$ Centre de Lutte Contre le Cancer Eugène Marquis, Rennes, France.

\title{
* Corresponding author:
}

Delphine Fessart - INSERM U1242 Université Rennes 1 -Centre de Lutte Contre le Cancer Eugène Marquis, Rue de la Bataille Flandres Dunkerque, 35042 Rennes, France. e-mail: delphine.fessart@yahoo.fr

Running Title: AGR2, tumor microenvironment and chemoresistance

Key Words: AGR2, PDI, Secretome, ER, Microenvironment, Cancer, Chemoresistance, Niche.

Abbreviations (if repeated at least 3 times)

AGR2 Anterior Gradient 2

CAFs cancer-associated fibroblasts

CRC colorectal carcinoma

CSC cancer stem cells 


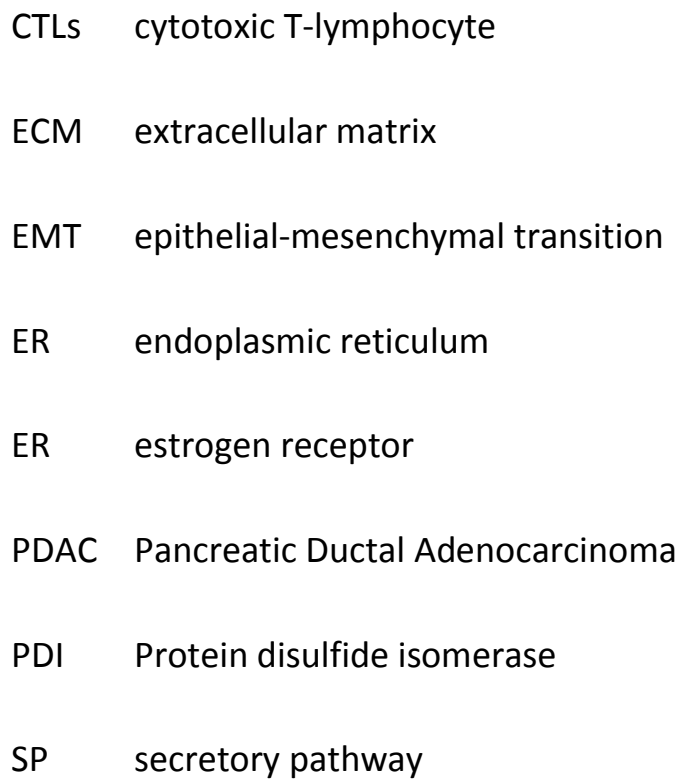

\section{$\underline{\text { Abstract }}$}

In recent years, the discovery of "tumor niche", a microenvironment that favors tumor development has changed our perspective of cancer. This microenvironment generated by the tumor cells itself and surrounding cells, is capable of providing essential elements for its growth. Consequently, the homeostasis of the Secretory Pathway (SP) has become an essential player in cancer development. The SP not only promotes cellular adaptation to protein misfolding due to oncogenic transformation or challenging tumor niche but also allows tumor cells to produce specific secretomes. This impacts tumor cells in cis- or trans- as well as stromal cells in the tumor niche. In this context, the Anterior GRadient 2 (AGR2) protein has been identified as a key player. AGR2 is a protein disulfide isomerase that resides in the Endoplasmic Reticulum (ER) and mediates the formation of disulfide bonds, catalyzes the cysteine-based redox reactions and assists the quality control of proteins. AGR2 not only plays an essential role in the homeostasis of the SP but also exerts pro-oncogenic gain-offunction due to its reported mislocalization in the tumor niche microenvironment. In this review, we summarize the dual role of AGR2, inside and outside the ER, on the tumor niche and its microenvironment. 


\section{Introduction}

For many years, a prevailing paradigm in cancer biology was that mutational aberrations in neoplastic cells are both necessary and sufficient for tumorigenesis. Hence, cancer was believed to be a cell-autonomous process. Thanks to the advances in biology and following fiascos of most cytotoxic chemotherapies and targeted therapies developed by the industry, the purely cellular cancer theory has been revised or even abandoned. Many researchers have adopted a new view that a tumor is not only composed of cancer cells but also an array of host cells. These include cancer-associated fibroblasts (CAFs), endothelial cells, cancer stem cells, immune cells and also the extracellular matrix (ECM) (Figure 1). Consequently, cancer is now considered as a systemic disease (as opposed to genetic or cellular) whose outcome depends essentially on the interactions of the tumor with the host specific environment, the tumor niche. Cancer cells recruit/activate nontransformed host cells and secreted proteins derived from both cancer and stromal cells to form a "Tumor Niche Secretome" (TNS). The tumor niche microenvironment (TNM) along with the TNS plays a critical role in cellular communications and thus favors tumor progression.

The proteins from TNS are mostly synthesized as protein-precursors, which contain $\mathrm{N}$ terminal signal peptides that direct them to the translocation apparatus of the Endoplasmic Reticulum (ER). These proteins are transported to the Golgi apparatus and subsequently, to the cell surface where they are liberated into the microenvironment by fusion of the Golgi-derived vesicles with the plasma membrane. This well-characterized protein secretion pathway is known as the classical Secretory Pathway (SP)(Mellman and Warren, 2000; Walter et al., 1984). Other evidence shows that, in addition to this mechanism, proteins can be exported by ER/Golgi-independent pathways through the so-called non-classical SP (Nickel, 2003). The secretion of pro-oncogenic factors such as growth factors, their associated receptors and of tumor microenvironment proteins confers specific dependency of the cancer cells towards the SP. This is well illustrated by the interaction of tumor cells with their environment (Bissell and Hines, 2011) (Figure 2). Indeed, tumorstroma interactions are controlled by cytokines, ECM proteins, matrix metalloproteases, integrins, and other contact proteins. Activated CAFs fuel proliferating tumors with glucose, fatty acids, and amino acids and modulate signaling of adjacent cancer cells by secreting various growth factors and 
cytokines (Lyssiotis and Kimmelman, 2017). By releasing a large number of Epithelial-mesenchymal transition (EMT)-inducing soluble factors and remodeling the ECM, they can also support cancer invasion and metastasis (Mezawa and Orimo, 2016). Endothelial cells, which line tumor blood vessels, are educated by cancer cells not only to produce pro-angiogenic factors but also to promote migration, metastasis, and evade anoikis (Maishi and Hida, 2017). Finally, tumor-infiltrating leukocytes are well-known to have a dual function in cancer (Whiteside, 2008). These include both effectors of adaptive immunity such as T lymphocytes, dendritic cells (DCs), and B cells, as well as mediators of innate immunity, including macrophages, polymorphonuclear leukocytes, and NK cells. They can eliminate cancer cells by presenting tumor-associated antigens on the MHC I and MHC II molecules, which consequently activate CD4 helper and CD8 cytotoxic T lymphocytes. However, infiltrating leukocytes can also promote tumor growth, metastasis, and chronic inflammation leading to the unfavorable outcomes for patients (Fridman et al., 2017). Cytokines and chemokines are released from a wide range of immune cells and are essential for communication with other immune (as well as non-immune) cells for inflammatory response coordination. Immunogenicity is antigenicity in an inflammatory milieu resulting in a successful tumoral response. Tumor niche cells are derived from normal host cells whose proteins are recognized as "self" molecules toward which the immune system is normally tolerant. In contrast, tumor niche cells are immunogenic to differing levels, and express a variety of tumor antigens (Yuan et al., 2016). Hence, the TNS consisting of factors derived from cancer, non-cancer cells and the surrounding stroma, plays a deterministic role in oncogenesis (Figure 2).

In the context of TNM, it is essential to better understand the underlying molecular mechanisms of tumor cell secretion and as such the human Anterior GRadient (AGR) family of proteins has been identified as key player in such functions (Chevet et al., 2013). Interestingly, AGR2, the prototypic member of the AGR family, was shown to play intracellular roles in the ER contributing to the SP (Chevet et al., 2013; Higa et al., 2011). This ER-resident protein is also secreted in cancer extracellularly AGR2 (eAGR2) and assumes novel signaling functions that exhibit prooncogenic and inflammation gain-of-functions in the TNM. Hence, AGR2 changes the TNM both by its ER (intracellular) and ECM (extracellular) functions and thus can control the nature of tumor niche. This review focuses on the diverse functions of AGR2 on the TNM, inside and outside the ER. We update AGR2's contribution to TNS in cancer progression, maintenance of cancer stem cells, 
development of chemoresistance, and discuss its potential as a key therapeutic target in many cancers.

\section{AGR2 and the Tumor Niche Secretome (TNS)}

AGR2,also known as PDIA17, XAG-2, hAG-2, an ER-resident protein mainly expressed in human epithelial cells, was assigned to the human Protein Disulfide Isomerase (PDI) family later than all other members (Shishkin et al., 2013). The PDI family has 21 proteins that are involved in numerous physiological and pathological processes (Shishkin et al., 2013). PDI proteins have an important structural feature in common, one or several thioredoxin-like domains and also similar intracellular localization - in the ER and/or in other membrane formations. In turn, the PDI family is considered as a part of the thioredoxin superfamily, various members of which contain a peculiar structural block or module ( 100-120 amino acid residues (aa)) in their polypeptide chains similar to a small thioredoxin protein including a 4aa specific motif (Cys-X-X-Cys) (Carvalho et al., 2006; Pan and Bardwell, 2006). AGR2 protein (and its gene) is the 17th member of the PDI family. The most convincing evidence was obtained after its inclusion in this family. Park et al., in their studies on mice, detected an unusual catalytic activity of AGR2: it interacted with mucin 2 (MUC2), a large glycoprotein rich in cysteine residues (Park et al., 2009). The cysteine residue in the thioredoxin-like domain of AGR2 was shown to form a hetero-disulfide bond with cysteine residues in the MUC2 molecule. This reaction is a prerequisite for secretion of MUC2 by intestinal epithelium cells that allows this protein perform its protective function in the intestine. Data on AGR2 localization in ER (intracellular AGR2, iAGR2) also correlate with their membership in the PDI family (Brychtova et al., 2011; Park et al., 2009). It was also shown that this feature of AGR2 is determined by a special Cterminal KTEL motif, the complete removal of which or aa substitutions in it affecting the protein functions (Gupta et al., 2012). Of note, in other members of the PDI family, the corresponding motifs (on function retention) have a different aa at position 2 (KDEL, KVEL, etc.). Mutants often hold the key to understand protein function, as they can interrupt cellular processes. Indeed, a panel of different mutants have been designed and reported in the literature to study AGR2 protein functions (See Toolbox1). The presence of a non-canonical ER retention motif (C-terminal KTEL) in AGR2 may be responsible for diverse trafficking of this molecule. However, recent data from Fessart et al, wherein the C-terminal KTEL motif is mutated to either KDEL, K172D, K172A or a STOP is inserted before the KTEL ( $\triangle$ KTEL) (See Toolbox1), suggests that ER-resident AGR2 protein is a secretory 
protein by itself and its extracellular function is independent of the KTEL motif. For the thioredoxinlike domain, data in literature demonstrate that the AXXA mutation affects the 3D structure of the protein making the SXXS inactive form more suitable for studying thioredoxin domain function (Fessart et al., 2016) (See Toolbox1). Finally, a protein named Reptin was identified by yeast twohybrid screen and validated as an AGR2-interacting protein in human cells. Reptin is a highly conserved member of the AAA+ family that can be found in numerous multiprotein complexes linked to transcription, DNA damage response, and nonsense-mediated RNA decay (Gallant, 2007; Jha and Dutta, 2009; Kim et al., 2006; Kim et al., 2005; Rashid et al., 2009). The ATP binding motifs of Reptin and the proposed substrate-binding loop of AGR2 are determinants that drive a specific complex between AGR2 and Reptin proteins. Therefore, different mutants have been made to characterize this interaction (Maslon et al., 2010)(See Toolbox1). Recently, AGR2 was found to dimerize (Gray et al., 2013) using specific mutants (See Toolbox1), however this complex was identified using an in vitro approach and the physiological relevance of these forms remain hypothetical.

The TNS has diverse consequences in the alteration of the TNM. First, it can lead to destabilization of the microenvironment architecture by ECM remodeling through changes in ECM component abundance or matrix metalloproteases (MMPs) expression; consequently, there is an increase in cancer dissemination and invasion (Bissell and Hines, 2011; Leung and Brugge, 2012). Second, by modulating messenger (i.e. chemokines, cytokines) or contact/adhesion protein abundance (i.e. membrane receptors, integrins), the TNS can trigger proliferation, migration and tumor angiogenesis (Paltridge et al., 2013). Third, it can lead to remodeling of the immune response in the tumor microenvironment (Paltridge et al., 2013). Fourth, it can modulate tumor immunogenicity by secreting tumor antigens into the tumor niche (Meeusen et al., 2017). In course of tumorigenesis, the regulation of protein homeostasis (proteostasis) is strongly involved in cellular maintenance. In particular, proteostasis of the SP and its first compartment, the ER, through TNS, play an active role in coping with intrinsic and extrinsic stresses to which transforming and tumor cells are exposed (Hetz et al., 2015). As part of this ER proteostasis control machinery, AGR2 plays significant role in the maintenance of ER homeostasis (Chevet et al., 2013; Higa et al., 2011). Moreover, similar to other PDI proteins (Lee and Lee, 2017), an extracellular AGR2 (eAGR2) is secreted in the ECM (Fessart et al., 2016) and exhibits pro-oncogenic gain-of-functions in the tumor 
microenvironment (Fessart et al., 2016). Hence, AGR2 changes the TNM both by its intracellular (ER) and extracellular (ECM) functions and thus controls, the nature of TNM. In this present part, we provide an in-depth description of AGR2 in the context of the tumor niche and how AGR2, through the TNS, might play a role in inflammation, immunogenicity, ECM remodeling and angiogenesis. We define the "AGR2 Tumor niche secretome" as the disruption of the TNM-mediated by AGR2 leading to the modulation of proteins such as growth factors and their associated receptors, extracellular matrix proteins, matrix metalloproteinases, inflammatory factors, integrins, immunogenic factors or pro-angiogenic factors.

Inflammation - In pancreatic cancer, it has been reported that AGR2 upregulates during inflammatory phase of Pancreatic ductal adenocarcinoma (PDAC) development (Dumartin et al., 2017) and that ER stress induced by AGR2 is associated with the acquisition of a pro-inflammatory phenotype. Furthermore, ER stress could be the mediator of an intercellular communication between epithelial and stromal cells (Dumartin et al., 2017). It is possible that paracrine mediators, including inflammatory mediators such as Reactive Oxygen Species (ROS), are involved in the intercellular communication between ER stress and AGR2 expression; however, this hypothesis remains to be addressed further. AGR2 link to inflammatory phenotype has also been reported in Agr2-/- mice; these mice showed increased Dextran Sodium Sulfate (DSS) susceptibility resulting in a more severe inflammatory phenotype than in control animals. This was further illustrated and explained by the lack of a typical inner mucus layer. In the tumor niche, tumor growth is aided by pro-inflammatory components that stimulate cell proliferation and reduce apoptosis, while antiinflammatory cytokines, contribute to tumor immune evasion. AGR2's participation in chronic inflammatory disorder as an inflammatory mediator might be involved in carcinogenesis (Zheng et al., 2006). Given the multifaceted role of inflammation in tumor niche, the involvement of the AGR2associated inflammatory signaling, the microenvironmental cross-talk and network needs to be further investigated. 
Immunogenicity - eAGR2 protein is expressed on the surface of colorectal cancer (CRC) and pancreatic cancer cell lines (Dumartin et al., 2011; Lee et al., 2012). Lee et al. identified AGR2 peptides based on human leukocyte antigen HLA-A*0201-binding motifs to determine whether eAGR2 can be used as a tumor-associated antigen in CRC (Lee et al., 2012). Among the peptides identified, two were shown to have potential to generate AGR2 peptide-specific cytotoxic Tlymphocyte (CTL) response. The CTLs had strong cytolytic activity against AGR2 peptide-pulsed cells and HLA-A*0201+ AGR2-positive CRC cell lines. AGR2 peptide-specific CTLs can be generated by stimulating autologous T cells with dendritic cell (DC) vaccinations pulsed with AGR2 peptide. These CTLs could be promising effector cells for immunotherapy in CRC as they are potent killer cells that are able to specifically and effectively lyse target cells. Thus, eAGR2 could act as a potentially important antigen for immunotherapy against CRC in clinical applications.

Remodeling of the Extracellular Matrix (ECM) - Recently, we have shown that eAGR2 is present in the ECM in lung cancer (Fessart et al., 2016). A similar role was described for PDIA3 in remodeling of the ECM during renal fibrosis (Dihazi, 2013). Moreover, high levels of eAGR2 could directly contribute to the tumorigenicity of cancer cells. Indeed, we have shown that restoration of eAGR2 in the microenvironment of AGR2-knockdown tumor cells reversed AGR2 depletion-induced on organoid-initiating frequency (a phenotype of transformed tumor cell). This indicates that the reduced organoid-initiating frequency induced by AGR2 depletion is most likely due to the reduction of eAGR2 in the tumor microenvironment (Fessart et al., 2016). This is consistent with the observation that conditioned media from cells silenced for AGR2 have a reduced ability to stimulate proliferation of pancreatic cancer cells (Ramachandran et al., 2008). We also demonstrated that eAGR2 drives lung tumor organoid formation, not by changing the redox nature of microenvironment but through its presence within the ECM. Together, these results highlight that eAGR2 represents a pro-oncogenic microenvironmental signaling protein and suggest an important role for eAGR2 in controlling ECM functionality, and thus in the remodeling of tumor niche.

Angiogenesis - Recently, eAGR2 was shown to be vascular endothelial growth factor receptor 2 (VEGFR2) stimulator in tumor progression by promoting angiogenesis (Jia et al., 2018). 
Mechanistically, eAGR2 directly interacted with Vascular endothelial growth factor A, resulting in enhancement of Vascular endothelial growth factor (VEGF)/VEGFR2 signaling to promote angiogenesis. eAGR2 interacts with Vascular endothelial growth factor A via its thioredoxin motif. Furthermore, eAGR2 has been shown to directly bind to VEGF and fibroblast growth factor 2, which are the major players in tumor angiogenesis, and enhances their activities (Guo et al., 2017). This enhancement is dependent on both the AGR2 self-dimerization region and the two signaling pathways. This activity can be blocked, at least partially, by a monoclonal antibody targeting the selfdimerization region in vitro and in vivo, suggesting that eAGR2 is a potential anti-tumor target, which influences the activities of multiple extracellular signaling molecules. iAGR2 was shown to stabilize p65 (Jia et al., 2018), which in turn transcriptionally regulates EMT gene patterns and plays a critical role in inducing an AGR2-facilitated aggressive phenotype. This AGR2 effect significantly blocked the antitumor activity of bevacizumab but not cabozantinib, providing evidence for anti-angiogenic agents in cancer therapy.

Collectively, the aforementioned information sheds light on the intricate tumor niche signaling networks in which AGR2 is involved. Thus, the specificity of AGR2 on the TNS and its impact in and out of the TNM is an interesting avenue to better characterize tumor niche and carcinogenesis.

\section{AGR2 and the cancer stem cells niche}

Cancer stem cells (CSCs) are tumor cells that can self-renew, with the capacity to initiate clonal tumor and a potential for clonal long-term repopulation (Plaks et al., 2015). CSCs cluster in niches within the tumor microenvironment, which helps preserving their phenotypic plasticity, protecting them from the immune system, and facilitating their metastatic potential. Tumor recurrence after chemotherapy or radiation remains a major obstacle for successful cancer treatment. An emerging concept in tumor biology is the CSC hypothesis which postulates that, in bulk tumors, the potential to grow and propagate is restricted to CSCs that can reinitiate the tumor 
even after a successful elimination of the primary tumor mass by surgery, chemotherapy or radiotherapy. The observation described in the section above suggests that AGR2 plays a key role in pro-survival, EMT, and self-renewal, which are characteristics of CSCs. Recently, it has been demonstrated in head and neck squamous-cell carcinoma, that the in vitro knockdown of AGR2 effectively decreased CSC markers such as SOX2, NANOG, and OCT4 (Ma et al., 2015). This study also showed that AGR2 promotes HNSCC progression and radio-chemotherapy by regulating CSC signaling pathway.

Considering the essential role of the TNM in regulating the CSC phenotype, AGR2 might be targeted to develop new therapeutic strategies against CSC niche microenvironments. However, the underlying mechanisms of AGR2 involvement in CSCs remain to be further explored.

\section{AGR2 and the chemoresistance niche}

Several studies have reported on drug resistant cells with or without stem cells characteristic after chemotherapy. There is emerging evidence suggesting that AGR2 expression is closely associated with hormone-dependent cancers and drug resistance. Clinical studies have shown that elevated expression of AGR2 predicted an unfavorable prognosis in breast cancer patients and mediated tamoxifen drug resistance as an estrogen agonist (Hengel et al., 2011; Hrstka et al., 2010). AGR2 could activate ER stress response genes (Dumartin et al., 2017) to stimulate cell proliferation of AGR2-negative pancreatic tumor cells and to enhance drug resistance (Ramachandran et al., 2008). Based on this, the development of anti-estrogen resistance in many tumors seriously limits the curative effect of fulvestrant chemotherapy. To explain the mechanism of drug resistance, it has been proposed that the addition of fulvestrant not only induces estrogen receptor entry into the nucleus but also leads AGR2 to relocate to the outer edge of the cells (Li et al., 2016). One hypothesis is that the fulvestrant induces conformation change and subsequent phosphorylation of estrogen receptor and releases the cytoplasmic estrogen receptor-bound AGR2. Therefore, without fulvestrant treatment, estrogen receptor-bound AGR2 is maintained in the cytoplasm. The newly synthesized AGR2 is then activated for secretion toward the cell membrane through the SP (Li et al., 2016). This is consistent with previous observations that AGR2 expression is directly regulated by estrogens and is associated with poor outcomes for estrogen receptor-positive breast cancer 
patients (Hengel et al., 2011; Hrstka et al., 2010; Innes et al., 2006). These findings indicate that estrogen receptor-dependent induction of AGR2 may also contribute to attenuation of p53 activity. p53 plays an important role in sensitivity to chemotherapy, as illustrated by many studies linking TP53 mutations to drug resistance (Chrisanthar et al., 2011; Dobes et al., 2014). One explanation could be that estrogen receptor-dependent signaling, including AGR2 upregulation, actively participates in attenuation of p53 activity and thereby contributes to the malignant phenotype in the early stages of breast cancer development. Interestingly, p53 is involved in the induced secretion of inhibitory factors (Van Meir et al., 1994) or the negative regulation of secreted pro-tumorigenic proteins (Khwaja et al., 2006). Thus, AGR2 by inhibiting p53 in tumors could be also change the TNM, by modulating secreted proteins whose extracellular levels are regulated by $\mathrm{p} 53$. Moreover, in vitro, it has been demonstrated that eAGR2 stimulated chemoresistance of PDAC cell lines (Ramachandran et al., 2008). We have also shown that eAGR2 is present in the ECM (Fessart et al., 2016). Several ECM proteins have been associated with resistance to chemotherapy (Netti et al., 2000). Thus, the effects of eAGR2 in the ECM in the tumor niche might explain the role of eAGR2 in chemoresistance.

Collectively, these data show that the interaction between the tumor microenvironment and AGR2 could result in the development of chemoresistance. These data also suggest that inhibition of eAGR2, in the tumor niche, could be a potential strategy to overcome chemoresistance.

\section{Other human AGR proteins}

The human Anterior Gradient family consists of three members, namely TXNDC12, AGR2 and AGR3, all belonging to the PDI family of proteins (Chevet et al., 2013).

Thioredoxin domain containing protein 12 (TXNDC12) or AGR1 (previously designated as ERp16, ERp18, ERp19, or hTLP19) is a 172 amino acid protein, localized in ER, which possesses a thioredoxin domain with an active motif (CGAC) and a C-terminal ER retention sequence (EDEL) (Jeong et al., 2008).TXNDC12 protein possesses an important role in disulfide bond formation in ER and is involved in cellular defense against prolonged ER stress (Jeong et al., 2008). TXNDC12 is ubiquitously expressed in all tissues (Galligan and Petersen, 2012). In Hela cells, its expression 
inhibits induction of apoptosis, while depletion enhanced apoptosis (Jeong et al., 2008). Additionally, using microarrays, TXNDC12 expression was detected in non-tumor lung tissue from lung adenocarcinoma patients, and could potentially associate with the patients' survival (Galvan et al., 2013). However, whether this protein is secreted in the TNM or not remains unknown.

Human anterior gradient protein 3 (AGR3; also known as AG3, PDIA18 or BCMP11) is the closest homologue of AGR2. Both molecules share $71 \%$ sequence identity. Structurally, AGR3 is a small single-domain member of the PDI family (Hatahet and Ruddock, 2009). Unlike most other members of the PDI family, AGR3 lacks the canonical CXXC active-site motif; instead, it has the CYQS sequence, suggesting that it is not involved in thiol-disulfide exchange in protein folding. AGR3 has an ER retention signal QSEL in C-terminal and is retained in the ER via its C-terminal motif (Raykhel et al., 2007). AGR3 is expressed in the ciliated cells of the airway epithelium and oviduct as well as in the stomach, prostate and liver (Bonser et al., 2015; Obacz et al., 2015). Unlike many other PDIfamily members, AGR3 is not induced by the unfolded protein response (Bonser et al., 2015), but it is upregulated by androgens and estrogens (Bu et al., 2013; Vaarala et al., 2012), and has been suggested to be a potential therapeutic target and/or a molecular marker for hormone-responsive breast cancer and prostate cancer (Bu et al., 2013; Fletcher et al., 2003; Garczyk et al., 2015; Gray et al., 2012; King et al., 2011; Obacz et al., 2015). A knockout mouse model implicated AGR3 in mucociliary clearance and in calcium-modulated ciliary beat frequency (Bonser et al., 2015). AGR3 was first characterized in breast cancer cell membranes and was shown to localize in secretory or endosome-like vesicles (Adam et al., 2003), suggesting a potential role of secreted form of AGR3. Moreover, extracellular AGR3 (eAGR3) is an attractive serum-based biomarker candidate. Indeed, Stefan Garczyk et al. have shown a significant increase of AGR3 protein serum concentration in sera from breast cancer patients (Garczyk et al., 2015). Despite these studies on its intracellular functions and the presence of eAGR3 in the serum from cancer patients, the physiological role of AGR3 in TNM has never been explored so far. 


\section{CONCLUSION}

Studies mainly on cell culture have clearly implicated the contribution of AGR2 in cancer cell proliferation, survival and therapeutic resistance. Now, there is emerging evidence that AGR2 not only arms cancer cells with pro-survival adaptive pathways to combat the host surveillance mechanisms, it also promotes a vicious feedback cycle where cancer cells and non-cancer cells in the tumor microenvironment cross stimulate each other leading to tumor progression and metastasis. Research into the involvement of AGR2 in the tumor microenvironment is at an early stage and offers exciting new opportunities for investigation. In this context, we propose an overview of the disruption of the TNS by AGR2 (Figure 3), where all microenvironmental proteins are assigned a possible origin from many types of cells within the TNS. Although our main focus is the eAGR2 secreted from cancer cells, we also included secretomes derived from non-cancer cells or as a result of tumor-host cell interactions. Cancer cells release eAGR2 which leads to the acquisition of a proinflammatory, angiogenesis and ECM remodeling phenotypes into the tumor niche. Moreover, eAGR2 by itself might also be an excellent candidate as a tumor-associated antigen for immunogenicity. Hence, the discovery that AGR2, a normally ER-resident protein, is secreted in the TNM with activities distinct from those of the ER localization changes the paradigm. Most tumor cells have the capacity to control the nature of their microenvironment through proteins secreted by

TNS. In an attempt to better explain the function of AGR2 in cancer, we previously proposed that the fundamental core role of AGR2 is to regulate ER's capacity to adapt to the microenvironment (Chevet et al., 2013). This ER buffering capacity of AGR2 can in turn have an impact on the nature of the cell secretome under physiological or pathological conditions. Consequently, disruption of the TNS by AGR2 leads to the modulation of proteins such as growth factors and their associated receptors, extracellular matrix proteins, matrix metalloproteinases, inflammatory factors, integrins, immunogenic factors or pro-angiogenic factors. Thus, AGR2 plays a dual role at the level of the tumor niche, first indirectly by affecting the control of the tumor cell secretome (AGR2 secretome) and second directly through gain-of-function extracellular roles into the tumor microenvironment (Figure 3). In conclusion, AGR2 plays an important role in tumorigenesis, remodeling tumor niche, chemoresistance and CSC phenotype though cancer and non-call cell-cell communications in the 
TNM (Figure 2 and 3). We are only at the beginning of understanding the potential implications of the AGR2 functions in the tumor niche.

\section{QUESTIONS AND FUTURE PERSPECTIVES}

In conclusion, the PDI protein, AGR2, is an ER-resident protein that plays intracellular roles contributing to the maintenance of proteostasis of the SP. Moreover, in cancer, the secreted AGR2 interacts with the ECM and plays extracellular pro-oncogenic roles independent of its ER function. In an attempt to better explain the function of AGR2 within the tumor niche, we proposed a fundamental role for AGR2 in the surrounding microenvironment of cancer cells: an intracellular function (ER) through the regulation of the TNS, and an extracellular function (ECM) in controlling the nature of TNM. Based on the unique and distinct roles of AGR2 that we have reviewed here, a number of basic and clinical research questions remain, including: (i) Are paracrine mediators, including inflammatory mediators, involved in the intercellular communication between ER stress and AGR2 expression and how? (ii) Is there a cross-talk between AGR2-associated inflammatory signaling, and the microenvironment? (iii) Does eAGR2 act as a potential antigen for immunotherapy against cancer? (iv) Is AGR2 a good target to develop new therapeutic strategies against CSC niche microenvironment? (v) What are the effects of eAGR2 in the ECM within the tumor niche that might explain the role of eAGR2 in chemoresistance ? (vi) Does the inhibition of eAGR2 in the TNM, could be a potential strategy to overcome chemoresistance? (vii) How the amount and content of TNS relies on the ability of AGR2 cell secretory pathway to adjust the protein folding and secretion demand of tumor cells? and (viii) Is the "AGR2 tumor niche secretome" that mediates its core functions in cell migration, growth or adhesion 'druggable'?

Answers to these fundamental questions would highlight the role of AGR2, and by extend other AGR proteins, in the TNM and give us an understanding of how the nature of the TNS provides advantages to cancer cell. 


\section{References}

Adam, P.J., R. Boyd, K.L. Tyson, G.C. Fletcher, A. Stamps, L. Hudson, H.R. Poyser, N. Redpath, M. Griffiths, G. Steers, A.L. Harris, S. Patel, J. Berry, J.A. Loader, R.R. Townsend, L. Daviet, P. Legrain, R. Parekh, and J.A. Terrett. 2003. Comprehensive proteomic analysis of breast cancer cell membranes reveals unique proteins with potential roles in clinical cancer. The Journal of biological chemistry. 278:6482-6489.

Bissell, M.J., and W.C. Hines. 2011. Why don't we get more cancer? A proposed role of the microenvironment in restraining cancer progression. Nature medicine. 17:320-329.

Bonser, L.R., B.W. Schroeder, L.A. Ostrin, N. Baumlin, J.L. Olson, M. Salathe, and D.J. Erle. 2015. The Endoplasmic Reticulum Resident Protein AGR3. Required for Regulation of Ciliary Beat Frequency in the Airway. American journal of respiratory cell and molecular biology. 53:536543.

Brychtova, V., B. Vojtesek, and R. Hrstka. 2011. Anterior gradient 2: a novel player in tumor cell biology. Cancer letters. 304:1-7.

Bu, H., M.R. Schweiger, T. Manke, A. Wunderlich, B. Timmermann, M. Kerick, L. Pasqualini, E. Shehu, C. Fuchsberger, A.C. Cato, and H. Klocker. 2013. Anterior gradient 2 and 3--two prototype androgen-responsive genes transcriptionally upregulated by androgens and by oestrogens in prostate cancer cells. The FEBS journal. 280:1249-1266.

Carvalho, A.P., P.A. Fernandes, and M.J. Ramos. 2006. Similarities and differences in the thioredoxin superfamily. Progress in biophysics and molecular biology. 91:229-248.

Chevet, E., D. Fessart, F. Delom, A. Mulot, B. Vojtesek, R. Hrstka, E. Murray, T. Gray, and T. Hupp. 2013. Emerging roles for the pro-oncogenic anterior gradient-2 in cancer development. Oncogene. 32:2499-2509.

Chrisanthar, R., S. Knappskog, E. Lokkevik, G. Anker, B. Ostenstad, S. Lundgren, T. Risberg, I. Mjaaland, G. Skjonsberg, T. Aas, E. Schlichting, H.E. Fjosne, A. Nysted, J.R. Lillehaug, and P.E. Lonning. 2011. Predictive and prognostic impact of TP53 mutations and MDM2 promoter genotype in primary breast cancer patients treated with epirubicin or paclitaxel. PloS one. 6:e19249.

Dihazi, H. 2013. Prognosis markers for metastatic renal cell carcinoma: quantitative proteomics approach. Expert review of proteomics. 10:21-24. 
Dobes, P., J. Podhorec, O. Coufal, A. Jureckova, K. Petrakova, B. Vojtesek, and R. Hrstka. 2014. Influence of mutation type on prognostic and predictive values of TP53 status in primary breast cancer patients. Oncology reports. 32:1695-1702.

Dong, A., D. Wodziak, and A.W. Lowe. 2015. Epidermal growth factor receptor (EGFR) signaling requires a specific endoplasmic reticulum thioredoxin for the post-translational control of receptor presentation to the cell surface. The Journal of biological chemistry. 290:80168027.

Dumartin, L., W. Alrawashdeh, S.M. Trabulo, T.P. Radon, K. Steiger, R.M. Feakins, M.P. di Magliano, C. Heeschen, I. Esposito, N.R. Lemoine, and T. Crnogorac-Jurcevic. 2017. ER stress protein AGR2 precedes and is involved in the regulation of pancreatic cancer initiation. Oncogene. 36:3094-3103.

Dumartin, L., H.J. Whiteman, M.E. Weeks, D. Hariharan, B. Dmitrovic, C.A. lacobuzio-Donahue, T.A. Brentnall, M.P. Bronner, R.M. Feakins, J.F. Timms, C. Brennan, N.R. Lemoine, and T. Crnogorac-Jurcevic. 2011. AGR2 is a novel surface antigen that promotes the dissemination of pancreatic cancer cells through regulation of cathepsins B and D. Cancer research. 71:7091-7102.

Fessart, D., C. Domblides, T. Avril, L.A. Eriksson, H. Begueret, R. Pineau, C. Malrieux, N. Dugot-Senant, C. Lucchesi, E. Chevet, and F. Delom. 2016. Secretion of protein disulphide isomerase AGR2 confers tumorigenic properties. eLife. 5.

Fletcher, G.C., S. Patel, K. Tyson, P.J. Adam, M. Schenker, J.A. Loader, L. Daviet, P. Legrain, R. Parekh, A.L. Harris, and J.A. Terrett. 2003. hAG-2 and hAG-3, human homologues of genes involved in differentiation, are associated with oestrogen receptor-positive breast tumours and interact with metastasis gene C4.4a and dystroglycan. British journal of cancer. 88:579-585.

Fridman, W.H., L. Zitvogel, C. Sautes-Fridman, and G. Kroemer. 2017. The immune contexture in cancer prognosis and treatment. Nature reviews. Clinical oncology. 14:717-734.

Gallant, P. 2007. Control of transcription by Pontin and Reptin. Trends in cell biology. 17:187-192.

Galligan, J.J., and D.R. Petersen. 2012. The human protein disulfide isomerase gene family. Human genomics. 6:6.

Galvan, A., E. Frullanti, M. Anderlini, G. Manenti, S. Noci, M. Dugo, F. Ambrogi, L. De Cecco, R. Spinelli, R. Piazza, A. Pirola, C. Gambacorti-Passerini, M. Incarbone, M. Alloisio, D. Tosi, M. Nosotti, L. Santambrogio, U. Pastorino, and T.A. Dragani. 2013. Gene expression signature of non-involved lung tissue associated with survival in lung adenocarcinoma patients. Carcinogenesis. 34:2767-2773. 
Garczyk, S., S. von Stillfried, W. Antonopoulos, A. Hartmann, M.G. Schrauder, P.A. Fasching, T. Anzeneder, A. Tannapfel, Y. Ergonenc, R. Knuchel, M. Rose, and E. Dahl. 2015. AGR3 in breast cancer: prognostic impact and suitable serum-based biomarker for early cancer detection. PloS one. 10:e0122106.

Gray, T.A., N.J. MacLaine, C.O. Michie, P. Bouchalova, E. Murray, J. Howie, R. Hrstka, M.M. Maslon, R. Nenutil, B. Vojtesek, S. Langdon, L. Hayward, C. Gourley, and T.R. Hupp. 2012. Anterior Gradient-3: a novel biomarker for ovarian cancer that mediates cisplatin resistance in xenograft models. Journal of immunological methods. 378:20-32.

Gray, T.A., E. Murray, M.W. Nowicki, L. Remnant, A. Scherl, P. Muller, B. Vojtesek, and T.R. Hupp. 2013. Development of a fluorescent monoclonal antibody-based assay to measure the allosteric effects of synthetic peptides on self-oligomerization of AGR2 protein. Protein science : a publication of the Protein Society. 22:1266-1278.

Guo, H., Q. Zhu, X. Yu, S.B. Merugu, H.B. Mangukiya, N. Smith, Z. Li, B. Zhang, H. Negi, R. Rong, K. Cheng, Z. Wu, and D. Li. 2017. Tumor-secreted anterior gradient-2 binds to VEGF and FGF2 and enhances their activities by promoting their homodimerization. Oncogene. 36:50985109.

Gupta, A., A. Dong, and A.W. Lowe. 2012. AGR2 gene function requires a unique endoplasmic reticulum localization motif. The Journal of biological chemistry. 287:4773-4782.

Hatahet, F., and L.W. Ruddock. 2009. Protein disulfide isomerase: a critical evaluation of its function in disulfide bond formation. Antioxidants \& redox signaling. 11:2807-2850.

Hengel, S.M., E. Murray, S. Langdon, L. Hayward, J. O'Donoghue, A. Panchaud, T. Hupp, and D.R. Goodlett. 2011. Data-independent proteomic screen identifies novel tamoxifen agonist that mediates drug resistance. Journal of proteome research. 10:4567-4578.

Hetz, C., E. Chevet, and S.A. Oakes. 2015. Proteostasis control by the unfolded protein response. Nature cell biology. 17:829-838.

Higa, A., A. Mulot, F. Delom, M. Bouchecareilh, D.T. Nguyen, D. Boismenu, M.J. Wise, and E. Chevet. 2011. Role of pro-oncogenic protein disulfide isomerase (PDI) family member anterior gradient 2 (AGR2) in the control of endoplasmic reticulum homeostasis. The Journal of biological chemistry. 286:44855-44868.

Hrstka, R., R. Nenutil, A. Fourtouna, M.M. Maslon, C. Naughton, S. Langdon, E. Murray, A. Larionov, K. Petrakova, P. Muller, M.J. Dixon, T.R. Hupp, and B. Vojtesek. 2010. The pro-metastatic protein anterior gradient-2 predicts poor prognosis in tamoxifen-treated breast cancers. Oncogene. 29:4838-4847. 
Innes, H.E., D. Liu, R. Barraclough, M.P. Davies, P.A. O'Neill, A. Platt-Higgins, S. de Silva Rudland, D.R. Sibson, and P.S. Rudland. 2006. Significance of the metastasis-inducing protein AGR2 for outcome in hormonally treated breast cancer patients. British journal of cancer. 94:10571065.

Jeong, W., D.Y. Lee, S. Park, and S.G. Rhee. 2008. ERp16, an endoplasmic reticulum-resident thioldisulfide oxidoreductase: biochemical properties and role in apoptosis induced by endoplasmic reticulum stress. The Journal of biological chemistry. 283:25557-25566.

Jha, S., and A. Dutta. 2009. RVB1/RVB2: running rings around molecular biology. Molecular cell. 34:521-533.

Jia, M., Y. Guo, D. Zhu, N. Zhang, L. Li, J. Jiang, Y. Dong, Q. Xu, X. Zhang, M. Wang, H. Yu, F. Wang, K. Tian, J. Zhang, C.Y.F. Young, H. Lou, and H. Yuan. 2018. Pro-metastatic activity of AGR2 interrupts angiogenesis target bevacizumab efficiency via direct interaction with VEGFA and activation of NF-kappaB pathway. Biochimica et biophysica acta. 1864:1622-1633.

Khwaja, F.W., P. Svoboda, M. Reed, J. Pohl, B. Pyrzynska, and E.G. Van Meir. 2006. Proteomic identification of the wt-p53-regulated tumor cell secretome. Oncogene. 25:7650-7661.

Kim, J.H., H.J. Choi, B. Kim, M.H. Kim, J.M. Lee, I.S. Kim, M.H. Lee, S.J. Choi, K.I. Kim, S.I. Kim, C.H. Chung, and S.H. Baek. 2006. Roles of sumoylation of a reptin chromatin-remodelling complex in cancer metastasis. Nature cell biology. 8:631-639.

Kim, J.H., B. Kim, L. Cai, H.J. Choi, K.A. Ohgi, C. Tran, C. Chen, C.H. Chung, O. Huber, D.W. Rose, C.L. Sawyers, M.G. Rosenfeld, and S.H. Baek. 2005. Transcriptional regulation of a metastasis suppressor gene by Tip60 and beta-catenin complexes. Nature. 434:921-926.

King, E.R., C.S. Tung, Y.T. Tsang, Z. Zu, G.T. Lok, M.T. Deavers, A. Malpica, J.K. Wolf, K.H. Lu, M.J. Birrer, S.C. Mok, D.M. Gershenson, and K.K. Wong. 2011. The anterior gradient homolog 3 (AGR3) gene is associated with differentiation and survival in ovarian cancer. The American journal of surgical pathology. 35:904-912.

Lee, E., and D.H. Lee. 2017. Emerging roles of protein disulfide isomerase in cancer. $B M B$ reports. 50:401-410.

Lee, H.J., C.Y. Hong, C.J. Jin, M.H. Kim, Y.K. Lee, T.N. Nguyen-Pham, H. Lee, B.C. Park, I.J. Chung, H.J. Kim, and J.J. Lee. 2012. Identification of novel HLA-A*0201-restricted epitopes from anterior gradient-2 as a tumor-associated antigen against colorectal cancer. Cellular \& molecular immunology. 9:175-183.

Leung, C.T., and J.S. Brugge. 2012. Outgrowth of single oncogene-expressing cells from suppressive epithelial environments. Nature. 482:410-413. 
Li, Z., Q. Zhu, H. Chen, L. Hu, H. Negi, Y. Zheng, Y. Ahmed, Z. Wu, and D. Li. 2016. Binding of anterior gradient 2 and estrogen receptor-alpha: Dual critical roles in enhancing fulvestrant resistance and IGF-1-induced tumorigenesis of breast cancer. Cancer letters. 377:32-43.

Lyssiotis, C.A., and A.C. Kimmelman. 2017. Metabolic Interactions in the Tumor Microenvironment. Trends in cell biology. 27:863-875.

Ma, S.R., W.M. Wang, C.F. Huang, W.F. Zhang, and Z.J. Sun. 2015. Anterior gradient protein 2 expression in high grade head and neck squamous cell carcinoma correlated with cancer stem cell and epithelial mesenchymal transition. Oncotarget. 6:8807-8821.

Maishi, N., and K. Hida. 2017. Tumor endothelial cells accelerate tumor metastasis. Cancer science. 108:1921-1926.

Maslon, M.M., R. Hrstka, B. Vojtesek, and T.R. Hupp. 2010. A divergent substrate-binding loop within the pro-oncogenic protein anterior gradient-2 forms a docking site for Reptin. Journal of molecular biology. 404:418-438.

Meeusen, E., E. Lim, and S. Mathivanan. 2017. Secreted Tumor Antigens - Immune Biomarkers for Diagnosis and Therapy. Proteomics. 17.

Mellman, I., and G. Warren. 2000. The road taken: past and future foundations of membrane traffic. Cell. 100:99-112.

Mezawa, Y., and A. Orimo. 2016. The roles of tumor- and metastasis-promoting carcinomaassociated fibroblasts in human carcinomas. Cell and tissue research. 365:675-689.

Netti, P.A., D.A. Berk, M.A. Swartz, A.J. Grodzinsky, and R.K. Jain. 2000. Role of extracellular matrix assembly in interstitial transport in solid tumors. Cancer research. 60:2497-2503.

Nickel, W. 2003. The mystery of nonclassical protein secretion. A current view on cargo proteins and potential export routes. European journal of biochemistry. 270:2109-2119.

Obacz, J., V. Brychtova, J. Podhorec, P. Fabian, P. Dobes, B. Vojtesek, and R. Hrstka. 2015. Anterior gradient protein 3 is associated with less aggressive tumors and better outcome of breast cancer patients. OncoTargets and therapy. 8:1523-1532.

Paltridge, J.L., L. Belle, and Y. Khew-Goodall. 2013. The secretome in cancer progression. Biochimica et biophysica acta. 1834:2233-2241.

Pan, J.L., and J.C. Bardwell. 2006. The origami of thioredoxin-like folds. Protein science : a publication of the Protein Society. 15:2217-2227.

Park, S.W., G. Zhen, C. Verhaeghe, Y. Nakagami, L.T. Nguyenvu, A.J. Barczak, N. Killeen, and D.J. Erle. 2009. The protein disulfide isomerase AGR2 is essential for production of intestinal mucus. 
Proceedings of the National Academy of Sciences of the United States of America. 106:69506955.

Plaks, V., N. Kong, and Z. Werb. 2015. The cancer stem cell niche: how essential is the niche in regulating stemness of tumor cells? Cell stem cell. 16:225-238.

Ramachandran, V., T. Arumugam, H. Wang, and C.D. Logsdon. 2008. Anterior gradient 2 is expressed and secreted during the development of pancreatic cancer and promotes cancer cell survival. Cancer research. 68:7811-7818.

Rashid, S., I. Pilecka, A. Torun, M. Olchowik, B. Bielinska, and M. Miaczynska. 2009. Endosomal adaptor proteins APPL1 and APPL2 are novel activators of beta-catenin/TCF-mediated transcription. The Journal of biological chemistry. 284:18115-18128.

Raykhel, I., H. Alanen, K. Salo, J. Jurvansuu, V.D. Nguyen, M. Latva-Ranta, and L. Ruddock. 2007. A molecular specificity code for the three mammalian KDEL receptors. The Journal of cell biology. 179:1193-1204.

Shishkin, S.S., L.S. Eremina, L.I. Kovalev, and M.A. Kovaleva. 2013. AGR2, ERp57/GRP58, and some other human protein disulfide isomerases. Biochemistry. Biokhimiia. 78:1415-1430.

Vaarala, M.H., P. Hirvikoski, S. Kauppila, and T.K. Paavonen. 2012. Identification of androgenregulated genes in human prostate. Molecular medicine reports. 6:466-472.

Van Meir, E.G., P.J. Polverini, V.R. Chazin, H.J. Su Huang, N. de Tribolet, and W.K. Cavenee. 1994. Release of an inhibitor of angiogenesis upon induction of wild type p53 expression in glioblastoma cells. Nature genetics. 8:171-176.

Walter, P., R. Gilmore, and G. Blobel. 1984. Protein translocation across the endoplasmic reticulum. Cell. 38:5-8.

Whiteside, T.L. 2008. The tumor microenvironment and its role in promoting tumor growth. Oncogene. 27:5904-5912.

Yuan, J., P.S. Hegde, R. Clynes, P.G. Foukas, A. Harari, T.O. Kleen, P. Kvistborg, C. Maccalli, H.T. Maecker, D.B. Page, H. Robins, W. Song, E.C. Stack, E. Wang, T.L. Whiteside, Y. Zhao, H. Zwierzina, L.H. Butterfield, and B.A. Fox. 2016. Novel technologies and emerging biomarkers for personalized cancer immunotherapy. Journal for immunotherapy of cancer. 4:3.

Zheng, W., P. Rosenstiel, K. Huse, C. Sina, R. Valentonyte, N. Mah, L. Zeitlmann, J. Grosse, N. Ruf, P. Nurnberg, C.M. Costello, C. Onnie, C. Mathew, M. Platzer, S. Schreiber, and J. Hampe. 2006. Evaluation of AGR2 and AGR3 as candidate genes for inflammatory bowel disease. Genes and immunity. 7:11-18. 
Figure 2 The Tumor Niche Secretome (TNS) controls the Tumor Niche Microenvironment (TNM).

TNS consists of factors derived from cancer, non-cancer cells and the surrounding stroma, and plays a deterministic role in oncogenesis. Cancer cells secrete: i) pro-angiogenic factors to stimulate the proliferation endothelial cells which in turn promote cancer survival and growth; ii) factors to activate cancer-associated fibroblastes; iii) proinflammatory signals; and iv) signals to modulate the properties of cancer stem cells. 


\section{Figure 3 Heterotypic overview of the AGR2 Tumor Niche Secretome.}

Schematic representation of AGR2 functions in the tumor niche microenvironment. All secreted proteins from the tumor niche may originate either from cancer cells or from associated stromal cells and their secretion may be triggered by paracrine or autocrine actions between them. AGR2 could be the mediator of an intercellular communication between both cancer and non-cancer cells via the release of eAGR2 and/or the disturbance of the TNS and thus leading to the acquisition of cancer features. eAGR2 protein is also found to be expressed on the surface of cancer cell lines, indicating that eAGR2 might be an excellent candidate as a tumor-associated antigen for immunogenicity. Furthermore, extracellular AGR2 has been reported to directly bind to VEGF and FGF2, which are the major players in tumor angiogenesis, and enhances their activities. eAGR2 has been also reported to be an important player in controlling ECM functionality and a proinflammatory phenotype, and most likely in the remodeling of tumor niche. It has also been proposed that AGR2 stimulate cell proliferation and to enhance drug resistance. 

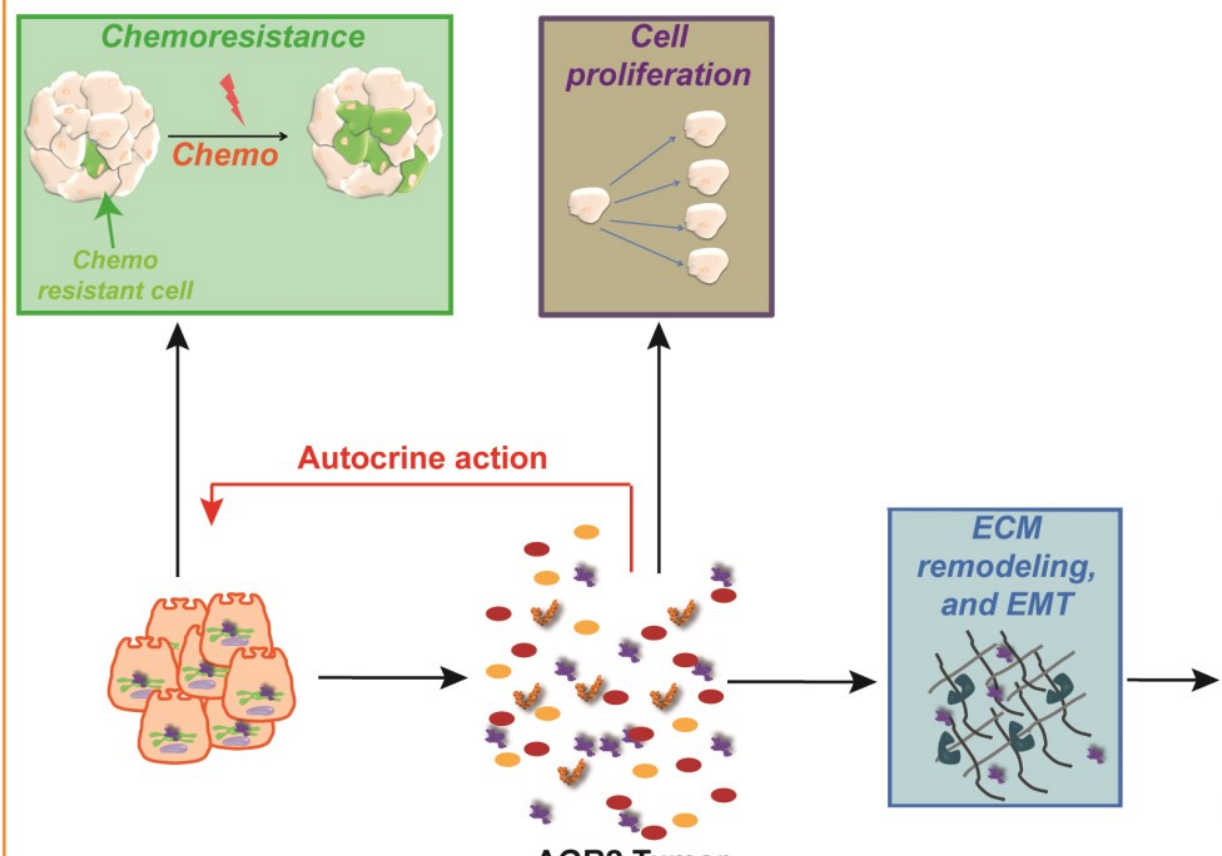

Metastasis

AGR2 Tumor Niche secretome
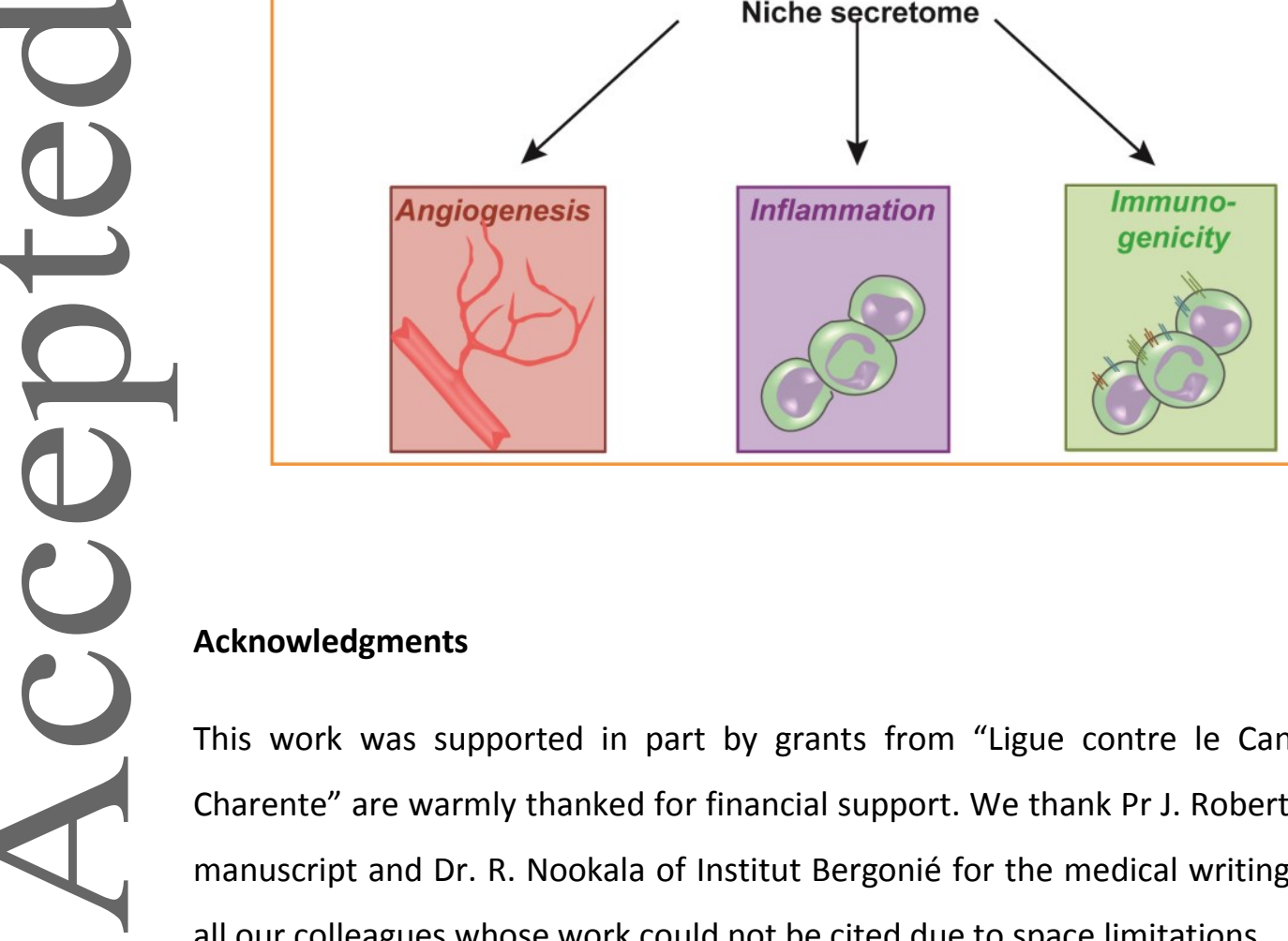

\section{Acknowledgments}

This work was supported in part by grants from "Ligue contre le Cancer- comités Gironde et Charente" are warmly thanked for financial support. We thank Pr J. Robert for critical reading of the manuscript and Dr. R. Nookala of Institut Bergonié for the medical writing service. We apologize to all our colleagues whose work could not be cited due to space limitations 
Toolbox 1. AGR2 and its mutants

\begin{tabular}{|c|c|c|}
\hline Mutations & Cellular Effects & References \\
\hline AGR2- $\triangle$ KTEL & $\begin{array}{l}\text { eAGR2 protein is secreted in the extracellular } \\
\text { medium, independently of its KTEL motif }\end{array}$ & $\begin{array}{l}\text { (Fessart et al., 2016; Gupta et } \\
\text { al., 2012) }\end{array}$ \\
\hline AGR2-K172D & $\begin{array}{l}\text { eAGR2 protein is secreted in the extracellular } \\
\text { medium, independently of its KTEL motif }\end{array}$ & (Fessart et al., 2016) \\
\hline AGR2-K172A & $\begin{array}{l}\text { eAGR2 protein is secreted in the extracellular } \\
\text { medium, independently of its KTEL motif }\end{array}$ & (Fessart et al., 2016) \\
\hline AGR2-KDEL & $\begin{array}{l}\text { eAGR2 protein is secreted in the extracellular } \\
\text { medium, independently of its KTEL motif, ER } \\
\text { localization }\end{array}$ & $\begin{array}{l}\text { (Fessart et al., 2016; Gupta et } \\
\text { al., 2012) }\end{array}$ \\
\hline AGR2-AXXA & $\begin{array}{l}\text { the inactive form of AGR2 thioredoxin-like } \\
\text { domain, mutant might be improperly folded }\end{array}$ & (Fessart et al., 2016) \\
\hline AGR2-SXXS & inactive form of $A G R 2$ thioredoxin-like domain & (Fessart et al., 2016) \\
\hline AGR2-C81A & $\begin{array}{l}\text { C81A active site mutant, and is able to form a } \\
\text { Reptin complex }\end{array}$ & $\begin{array}{l}\text { (Dong et al., 2015; Maslon et } \\
\text { al., 2010) }\end{array}$ \\
\hline AGR2-F104A & $\begin{array}{l}\text { did not reduce the stability of the Reptin-AGR2 } \\
\text { complex }\end{array}$ & (Maslon et al., 2010) \\
\hline AGR2-Y104A & $\begin{array}{l}\text { AGR2F104A mutant protein completely loses the } \\
\text { ability to bind Reptin indicating that the F104 } \\
\text { residue forms an important contact point for } \\
\text { Reptin in the context of the full-length protein } \\
\text { complex. }\end{array}$ & (Maslon et al., 2010) \\
\hline AGR2- $\Delta 45$ & stable dimer, active in binding to Reptin & (Gray et al., 2013) \\
\hline AGR2-E60A & monomeric & (Gray et al., 2013) \\
\hline
\end{tabular}




\begin{tabular}{|c|c|c|}
\hline AGR2- $\Delta$ KTEL & $\begin{array}{l}\text { eAGR2 protein is secreted in the extracellular } \\
\text { medium, independently of its KTEL motif }\end{array}$ & $\begin{array}{l}\text { (Fessart et al., 2016; Gupta et } \\
\text { al., 2012) }\end{array}$ \\
\hline AGR2-K172D & $\begin{array}{l}\text { eAGR2 protein is secreted in the extracellular } \\
\text { medium, independently of its KTEL motif }\end{array}$ & (Fessart et al., 2016) \\
\hline AGR2-K172A & $\begin{array}{l}\text { eAGR2 protein is secreted in the extracellular } \\
\text { medium, independently of its KTEL motif }\end{array}$ & (Fessart et al., 2016) \\
\hline AGR2-KDEL & $\begin{array}{l}\text { eAGR2 protein is secreted in the extracellular } \\
\text { medium, independently of its KTEL motif, ER } \\
\text { localization }\end{array}$ & $\begin{array}{l}\text { (Fessart et al., 2016; Gupta et } \\
\text { al., 2012) }\end{array}$ \\
\hline AGR2-AXXA & $\begin{array}{l}\text { the inactive form of AGR2 thioredoxin-like } \\
\text { domain, mutant might be improperly folded }\end{array}$ & (Fessart et al., 2016) \\
\hline AGR2-SXXS & inactive form of AGR2 thioredoxin-like domain & (Fessart et al., 2016) \\
\hline AGR2-C81A & $\begin{array}{l}\text { C81A active site mutant, and is able to form a } \\
\text { Reptin complex }\end{array}$ & $\begin{array}{l}\text { (Dong et al., 2015; Maslon et } \\
\text { al., 2010) }\end{array}$ \\
\hline AGR2-F104A & $\begin{array}{l}\text { did not reduce the stability of the Reptin-AGR2 } \\
\text { complex }\end{array}$ & (Maslon et al., 2010) \\
\hline AGR2-Y104A & $\begin{array}{l}\text { AGR2F104A mutant protein completely loses the } \\
\text { ability to bind Reptin indicating that the F104 } \\
\text { residue forms an important contact point for } \\
\text { Reptin in the context of the full-length protein } \\
\text { complex. }\end{array}$ & (Maslon et al., 2010) \\
\hline AGR2- $\triangle 45$ & stable dimer, active in binding to Reptin & (Gray et al., 2013) \\
\hline AGR2-E60A & monomeric & (Gray et al., 2013) \\
\hline
\end{tabular}

